\title{
Heteroepitaxy of Co-based Heusler compound/muscovite for flexible spintronics
}

Yi-Cheng Chen ${ }^{1,2}$, Min Yen ${ }^{1}$, Yu-Hong Lai ${ }^{1}$, Anastasios Markou², Liguo Zhang ${ }^{2}$, Yi-

Ying Chin ${ }^{3}$,Hong-Ji Lin ${ }^{4}$, Chien-Te Chen ${ }^{4}$, Claudia Felser ${ }^{2}$, Ying-Hao Chü, 1,5*

${ }^{1}$ Department of Materials Science and Engineering, National Chiao Tung University,

Hsinchu 30010, Taiwan

${ }^{2}$ Max Planck Institute for Chemical Physics of Solids, Dresden 01187, Germany

${ }^{3}$ Department of Physics, National Chung Cheng University, Chiayi 62102, Taiwan

${ }^{4}$ National Synchrotron Radiation Research Center, Hsinchu 30010, Taiwan

${ }^{5}$ Center for Emergent Functional Matter Science, National Chiao Tung University,

Hsinchu 30010, Taiwan

${ }^{6}$ Institue of Physics, Academia Sinica, Taipei 11529, Taiwan

\section{Corresponding Author}


* Correspondence to: yhc@,nctu.edu.tw

In Heusler compound, homogeneity plays an important role. The separation of each element would determine the intrinsic properties of the Heusler compound. For further investigation of the element spatial distribution in the $\mathrm{CMG} /$ muscovite, the EDS mapping was applied. In Figure S1, the spots with different colors indicate the count rate intensity of specific element. Co, Mn and Ga were detected in the same area of the thin film. The uniform distribution of the spots in the figure represents the welldistributed elements. The result shows the good homogeneity of $\mathrm{Co}, \mathrm{Mn}$ and $\mathrm{Ga}$ in CMG/muscovite heteroepitaxy.
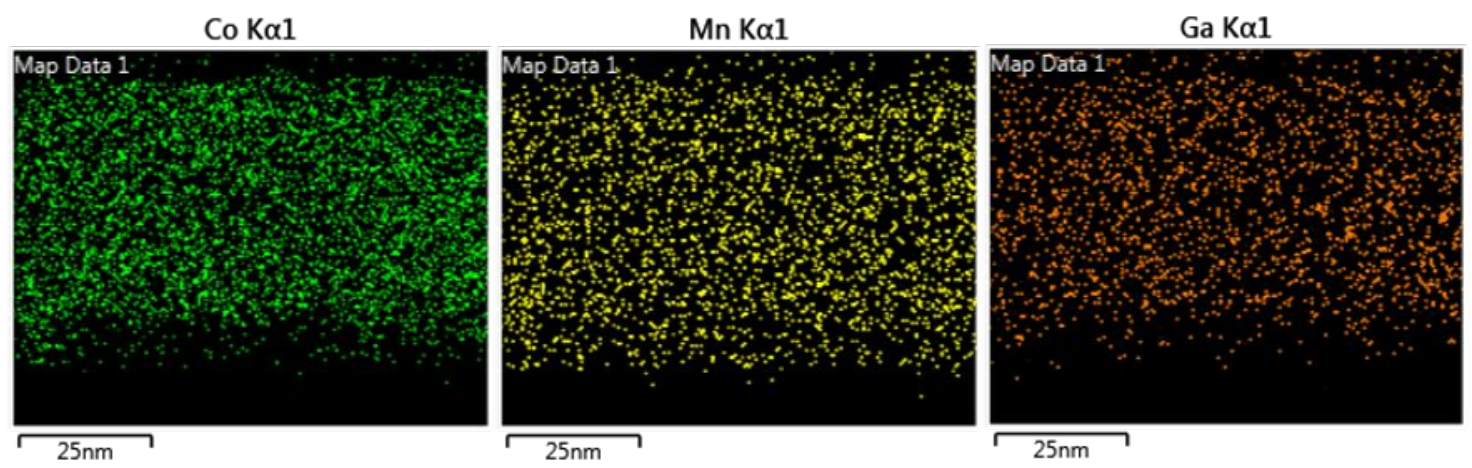

Figure S1

Elemental mapping of Co (Green), Mn (yellow) and Ga (orange). 